\title{
3D-DWT Improves Prediction of AD and MCI
}

\author{
Shuihua WANG, Yi CHEN, Yudong ZHANG, \\ School of Computer Science and Technology \\ Nanjing Normal University \\ Nanjing, Jiangsu 210023, China \\ Email: zhangyudong@njnu.edu.cn
}

\author{
Elizabeth LEE \\ Department of Engineering Technology \\ Chattanooga State Community College \\ Chattanooga, TN 37406, USA
}

\author{
Zhengchao DONG \\ Translational Imaging Division \& MRI Unit \\ Columbia University and New York State Psychiatric \\ Institute \\ New York, NY 10032, USA
}

\author{
Preetha PHILLIPS \\ School of Natural Sciences and Mathematics \\ Shepherd University \\ Shepherdstown, West Virginia, 25443, USA
}

\begin{abstract}
In order to predict subjects with Alzheimer's disease (AD) and mild cognitive impairment (MCI) from normal elder controls (NC) more accurately, we compared two different kinds of discrete wavelet transform (DWT) based feature extraction techniques: multi-slice 2D-DWT and 3D-DWT. The dataset contained the magnetic resonance (MR) images of 178 subjects consisting of 97 NCs, 57 MCIs, and 24 ADs. We constructed two multiclass kernel support vector machine (MKSVM) classifiers based on multislice 2D-DWT features and 3D-DWT features, respectively. 5-fold cross validation was employed to obtain the out-of-sample estimate. Each classifier runs 10 times. Welch's t-test showed that the mean of the overall accuracy by 3D-DWT was higher than that of multislice 2DDWT, and the difference was statistically significant $(p=0.0146)$.
\end{abstract}

Keyword-Discrete Wavelet Transform; Magnetic Resonance Imaging; Multiclass Kernel Support Vector Machine; Cross Validation; Alzheimer's Disease; Mild Cognitive Impairment.

\section{Introduction}

Alzheimer's disease (AD) is a type of dementia. Its symptoms develop slowly and worsen over time, and may become severe enough to interfere with daily life, and lead to death. As the world evolves into an aging society, the burdens by $\mathrm{AD}$ on the society increase significantly [1]. The US healthcare system is predicted to cost $\$ 1$ trillion per year by 2050 . Therefore, early detection of $\mathrm{AD}$ is beneficial for the society. Mild cognitive impairment (MCI) is frequently seen as a prodromal stage of AD. In a word, detecting AD and MCI accurately and effectively from normal elder healthy people is of great importance.

Magnetic resonance imaging (MRI) [2-4] is a new tool that plays an important role in classifying among AD subjects, MCI subjects, and normal elder controls (NC). Recent literatures [510] have shown MR images based machine-learning is an emerging and powerful method for automatic prediction of presence of $\mathrm{AD}$ and MCI. However, aforementioned studies treated 3D brain as multislice 2D images, and employed 2D discrete wavelet transform (2D-DWT) techniques for each slice of image. This did not take into account the compressibility of the slice-dimension of the 3D brain, so the wavelet coefficients along the slice direction have redundant information.

In this study, we suggested using 3D-DWT based feature extraction technique instead, and compared it with multislice 2D-DWT technique. The results show the superiority of 3DDWT to multislice 2D-DWT in terms of overall classification accuracy.

\section{Data Procurement}

We download the public dataset from Open Access Series of Imaging Studies (OASIS) (url http://www.oasis-brains.org/) We choose the cross-sectional dataset corresponding to the MRI scan of individuals at a single point in time. The OASIS dataset consists of 416 subjects aged 18 to 96 . We exclude subjects under 60 years old, and then pick up 178 subjects (97 NCs, 57 MCIs, and 24 ADs) from the rest subjects. For each subject, 3 or 4 individual T1-weighted 3D MR images obtained in a single scan session are included. The subjects are all right-handed, and include both men and women.

The clinical dementia rating (CDR) was interpreted as the target data. Subjects with CDR of $0,0.5$, and 1 are considered NC, MCI, and AD, respectively [11]. Eight additional features involve gender (M/F), age, education, and socioeconomic status (SES), the mini-mental state examination (MMSE), the estimated total intracranial volume (eTIV) $\left(\mathrm{mm}^{3}\right)$, atlas scaling factor (ASF), and normalized whole brain volume (nWBV).

\section{Methodology}

The 3D-DWT is an excellent feature extraction (FE) technique that expands the 2D-DWT to 3D for treating volume data. 2D- 
DWT decomposes data in row and column direction. Further, the 3D-DWT decomposes data in row, column, and slice direction. Mathematically, 3D-DWT with separable wavelets can be regarded as the process of applying 1D-DWT on each vector along $z$-axis that share $x$-axis and $y$-axis coordinates after applying 2D-DWT for all comprising planes, pixels in which are located in the same $z$-axis positions.

Considering $\mathcal{V}$ represents a 3D volumetric data, and $\mathcal{V}(x$, $y, z)$ as a voxel located at the coordinate $(x, y, z)$. We utilize energy $\boldsymbol{e}$, variance $\boldsymbol{v}$, and Shannon entropy $\boldsymbol{s}$ as the descriptors of the volume data $\mathcal{V}$. They are defined as

$$
\begin{gathered}
e=\sum_{x} \sum_{y} \sum_{z} \mathcal{V}^{2}(x, y, z) \\
v=\sum_{x} \sum_{y} \sum_{z}(\mathcal{V}(x, y, z)-\overline{\mathcal{V}})^{2}
\end{gathered}
$$

where $\overline{\mathcal{V}}$ represents the mean value of volume data $\mathcal{V}$.

$$
s=-\sum_{x} \sum_{y} \sum_{z} \mathcal{V}^{2}(x, y, z) \log \left(1+\mathcal{V}^{2}(x, y, z)\right)
$$

The volumetric MR images of each individual are of dimension 176 × 208 x $176=6,443,008$. We performed 3-level 3D-DWT of "db2" wavelet on each 3D MR image, obtaining 8 $+7+7=22$ subbands ( 8 subbands of level 3, 7 subbands of level 2, and 7 subbands of level 1). For each subband, the triplet $(\boldsymbol{e}, \boldsymbol{v}, \boldsymbol{s})$ is extracted. Therefore, totally 66 volumetric features are obtained from the volume data for each subject. Meanwhile, for the multislice 2D-DWT technique, we select five key slices along the axial direction, and performed 3-level 2D-DWT of "db2" on each image. We obtain $4+3+3=10$ subbands. The same triplet is extracted for each subband. In total, $10 \times 3 \times 5=$ 150 features are obtained.

Remembering there are 8 additional features for each subject. Although $66+8=74$ features of 3D-DWT and $150+8$ $=158$ features of multislice 2D-DWT are not a burden for latest computers, they may complicate the subsequent corresponding classifier and increase computational resources. PCA is chosen, since it effectively reduces the dimensionality of the data and therefore reduces the computational cost of analyzing new data [12-14]. The threshold is set to select enough PC numbers that can explain at least $95 \%$ of the total variance.
Finally, we submitted the reduced feature to the support vector machine (SVM) for automatically classification among $\mathrm{AD}, \mathrm{NC}$ and MCI. Kernel technique [15-17] is used to further improve the performance of SVM, and we choose the radial basis function (RBF) kernel. Moreover, Multiclass technique is used since it is a three-class problem, and we choose the Winner-Takes-All (WTA) technique [10]. We dub the classifier as multiclass kernel SVM (MKSVM). In addition, the weights/biases of the classifier are trained by Sequential Minimal Optimization (SMO) method [18].

$K$-fold Cross-validation is employed to obtain the out-ofsample error [19-21]. $K$ is assigned with a value of 5 . For $k$ th run of the 5-fold CV, we take $k$ th fold as the test set, and other four folds as the training set. The Confusion Matrix (CM) of each run is recorded, and the overall classification accuracy (CA) is calculated.

Above procedure repeats 10 times independently, each time 5 folds partitioned randomly. Welch's t-test is conducted to give quantified proof whether 3D-DWT is better than multislice 2D-DWT. The null hypothesis is that the CAs of two methods are with equal means, without assuming they have equal variances. The alternative hypothesis is they are with unequal means. The t-test is carried out at $95 \%$ confidence interval.

\section{Experiments and Results}

The programs were in-house developed using Matlab 2014a, and run on IBM laptop with $3 \mathrm{GHz}$ Intel i3 dual-processor and 8GB RAM. 5-fold cross validation is employed to get the outof-sample estimate.

Table 1 compared the decomposition results of 3D-DWT with multi-slice 2D-DWT of a prescribed subject. The ISOvalue of $3 \mathrm{D}$ rendering is assigned with a value of 0.01 . The $3 \mathrm{D}$ textures in Table 1 are clearly visible in Level 1, and they turn coarser as the spatial resolution decreases, attributed to the fact that a low-pass subband dyadically cut-off into eight higherlevel subbands. We set the level of decomposition to 3 , since too low resolution contains little information.

Table 1 FE Comparison of FE method of a specific subject (2D-DWT shows only 1 of 5 key slices)

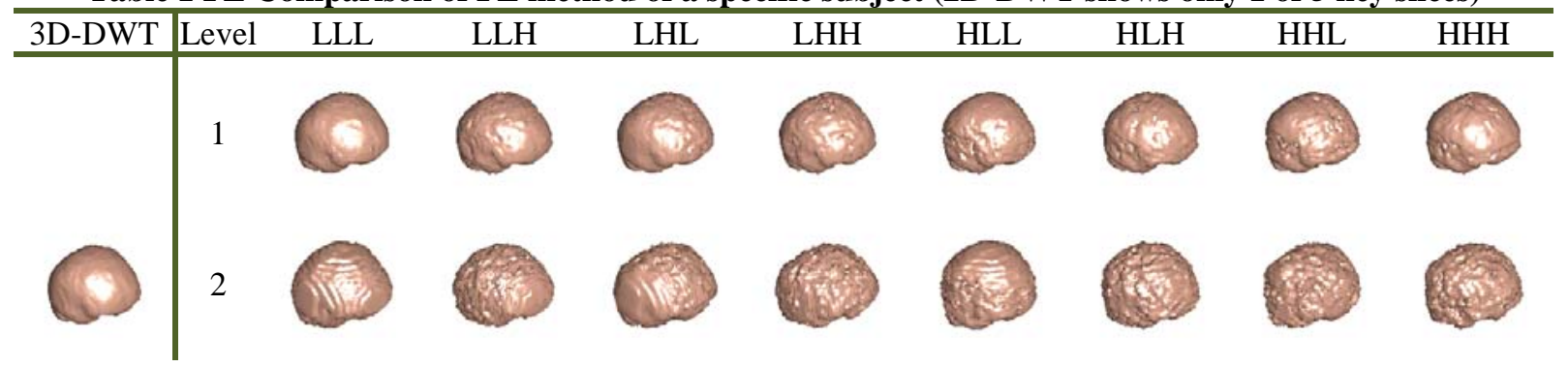




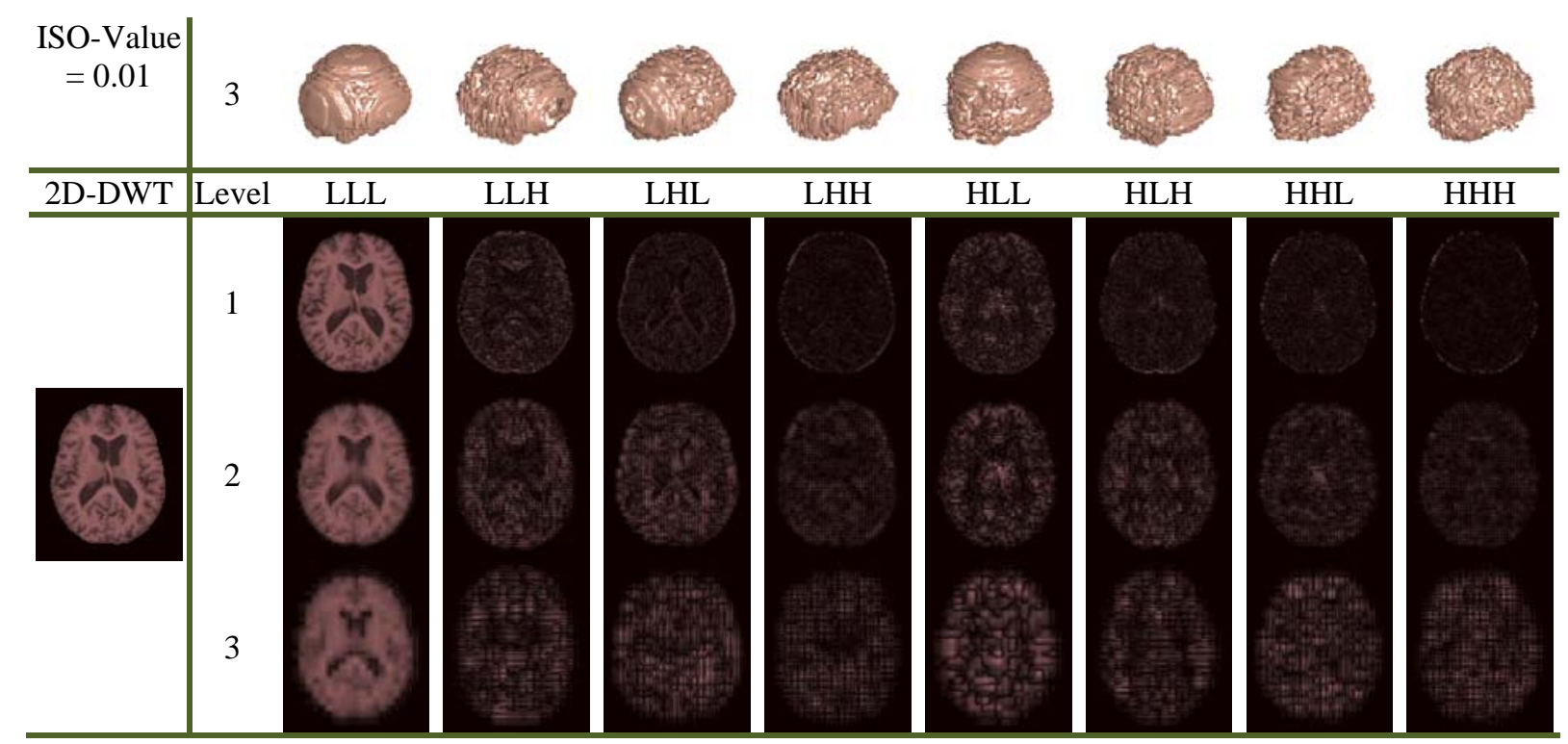

Table 2 gives the Welch's t-test results. The average CA of 3D-DWT is $79.8 \%$, higher than the average CA of multi-slice 2D-DWT of $77.0 \%$. Furthermore, the difference is statistically significant $(\mathrm{p}=0.0146)$.

Table 2 Results of Welch's t-test $(p=0.0146)$

\begin{tabular}{ll}
\hline Method & CA \\
\hline 3D-DWT & $0.798 \pm 0.022$ \\
Multislice 2D-DWT & $0.770 \pm 0.024$ \\
\hline
\end{tabular}

\section{Conclusions and Future Research}

In this study, we compared 3D-DWT with multi-slice 2DDWT in the application of prediction of AD and MCI from NC. We used the MKSVM classifier. The results show that the overall accuracies of three classes by 3D-DWT are higher than multislice 2D-DWT, and the difference is statistically significant $(p=0.0146)$. The future tentative work will center in comparison of 3D-DWT with other 3D features.

\section{ACKNOWLEDGMENT}

The work is supported by NSFC (No. 610011024), NJNU Research Foundation of Talented Scholars (No. 2013119XGQ0061, No. 2014119XGQ0080), and Program of Natural Science Research of Jiangsu Higher Education Institutions of China (No. 14KJB520021). Besides, the authors express their gratitude of the OASIS dataset that comes from NIH grants P50AG05681, P01 AG03991, R01 AG021910, P50 MH071616, U24 RR021382 and R01 MH56584.

\section{REFERENCES}

1. Hahn, K., et al., Selectively and progressively disrupted structural connectivity of functional brain networks in Alzheimer's disease - Revealed by a novel framework to analyze edge distributions of networks detecting disruptions with strong statistical evidence. NeuroImage, 2013. 81(0): p. 96-109.

2. Goh, S., et al., Mitochondrial dysfunction as a neurobiological subtype of autism spectrum disorder: Evidence from brain imaging. JAMA Psychiatry, 2014. 71(6): p. 665-671.

3. Zhang, Y., B. Peterson, and Z. Dong, A support-based reconstruction for SENSE MRI. Sensors, 2013. 13(4): p. 4029-40.

4. Dong, Z., et al., Improving the spectral resolution and spectral fitting of $1 H$ MRSI data from human calf muscle by the SPREAD technique. NMR in Biomedicine, 2014. 27(11): p. 1325-1332.

5. Nanthagopal, A.P. and R.S. Rajamony, Classification of benign and malignant brain tumor CT images using wavelet texture parameters and neural network classifier. Journal of Visualization, 2013. 16(1): p. 19-28.

6. Saritha, M., K. Paul Joseph, and A.T. Mathew, Classification of MRI brain images using combined wavelet entropy based spider web plots and probabilistic neural network. Pattern Recognition Letters, 2013. 34(16): p. 2151-2156.

7. Zhang, Y., S. Wang, and Z. Dong, Classification of Alzheimer Disease Based on Structural Magnetic Resonance Imaging by Kernel Support Vector Machine Decision Tree. Progress In Electromagnetics Research, 2014. 144: p. 171-184. 
8. Chaplot, S., L.M. Patnaik, and N.R. Jagannathan, Classification of magnetic resonance brain images using wavelets as input to support vector machine and neural network. Biomedical Signal Processing and Control, 2006. 1(1): p. 86-92.

9. El-Dahshan, E.-S.A., T. Hosny, and A.-B.M. Salem, Hybrid intelligent techniques for MRI brain images classification. Digital Signal Processing, 2010. 20(2): p. 433-441.

10. Zhang, Y. and L. Wu, An Mr Brain Images Classifier via Principal Component Analysis and Kernel Support Vector Machine. Progress In Electromagnetics Research, 2012. 130: p. 369-388.

11. Marcus, D.S., et al., Open Access Series of Imaging Studies (OASIS): cross-sectional MRI data in young, middle aged, nondemented, and demented older adults. J Cogn Neurosci, 2007. 19(9): p. 1498-507.

12. Kalbkhani, H., M.G. Shayesteh, and B. Zali-Vargahan, Robust algorithm for brain magnetic resonance image (MRI) classification based on GARCH variances series. Biomedical Signal Processing and Control, 2013. 8(6): p. 909-919.

13. Arjmandi, M.K. and M. Pooyan, An optimum algorithm in pathological voice quality assessment using waveletpacket-based features, linear discriminant analysis and support vector machine. Biomedical Signal Processing and Control, 2012. 7(1): p. 3-19.
14. Zhang, Y., et al., Fruit classification using computer vision and feedforward neural network. Journal of Food Engineering, 2014. 143(0): p. 167-177.

15. Xiao, Y., et al., Two methods of selecting Gaussian kernel parameters for one-class SVM and their application to fault detection. Knowledge-Based Systems, 2014. 59(0): p. 75-84.

16. Aich, U. and S. Banerjee, Modeling of EDM responses by support vector machine regression with parameters selected by particle swarm optimization. Applied Mathematical Modelling, 2014. 38(11-12): p. 2800-2818.

17. Zhang, Y., et al., An improved quality guided phase unwrapping method and its applications to MRI. Progress In Electromagnetics Research, 2014. 145: p. 273-286.

18. Guo, D.L., et al., Improved Radio Frequency Identification Indoor Localization Method via Radial Basis Function Neural Network. Mathematical Problems in Engineering, 2014.

19. Lee, W., B. Park, and K. Han, Classification of diffusion tensor images for the early detection of Alzheimer's disease. Computers in Biology and Medicine, 2013. 43(10): p. 1313-1320.

20. Römer, C., et al., Robust fitting of fluorescence spectra for pre-symptomatic wheat leaf rust detection with Support Vector Machines. Computers and Electronics in Agriculture, 2011. 79(2): p. 180-188.

21. Zhang, Y., et al., A hybrid method for MRI brain image classification. Expert Systems with Applications, 2011. 38(8): p. 10049-10053. 